\title{
PROGNOSIS IN SUPRACONDYLAR FRACTURES
}

\author{
T. S. Mann, Perth, Scotland \\ From the Western Infirmary, and the Royal Hospital for Sick Children, Glasgow
}

The purpose of this paper is to analyse the causes of permanent disability after supracondylar fractures of the humerus in children. By so doing $I$ hope to show what degree of residual displacement can be accepted without detriment to the patient, and that which must be corrected to obtain an elbow of normal appearance and a full range of movement.

A survey of the literature reveals many conflicting attitudes towards this fracture. It is agreed that accurate reduction is desirable, but there is variance of opinion about the cause of permanent deformity. Sandegaard (1943) analysed a large number of supracondylar fractures and concluded that anatomical reduction was important; he recommended that all severely displaced fractures should be treated by open reduction and nail fixation. On the other hand Siris (1939) after reviewing 330 fractures suggested that open operation was not justified because of the uniformly good results of closed manipulation, even when accurate reduction had not been obtained. Attenborough (1953) described functionally good results after extensive remodelling in four patients in whom the fractures had been left substantially unreduced.

It is agreed that cubitus varus is an undesirable result but its cause is still vaguely understood. It has been suggested that the injury may cause a differential growth of bone and that varus deformity follows overgrowth of the lateral metaphysis (Brewster and Karp 1940). But most authors attribute deformity to a mechanical effect caused by an inaccurately reduced fracture.

Formerly cubitus varus was thought to be the result of a residual displacement of the distal fragment in a medial direction (Siris 1939), but recently rotation and angulation have been considered. Many believe that the sole cause of a varus deformity is incomplete correction of medial rotation (Laurence 1956, French 1959), and this concept has been used to advocate novel forms of treatment (Høyer 1952, Madsen 1955). In contrast, retrospective studies of patients with cubitus varus (King and Secor 1951, Smith 1960) suggested that medial angulation is the relevant deformity.

In general much attention is given in the literature to individual methods of treatment of supracondylar fractures; but there is little to assist in predicting the outcome of a fracture which is not perfectly reduced. Most papers attempt to draw statistical comparisons between series of cases: few attempt to read lessons from careful study of the individual patient.

\section{PRESENT REVIEW}

During the two years 1958-59 eighty-five children with supracondylar fractures of the humerus attended the Royal Hospital for Sick Children, Glasgow. Sixty-two of these fractures did not require manipulation, or had only slight displacement which was easily reduced; all these patients had excellent results. It was only when the original displacement was more than one diameter and when contact between the broken bone ends had been lost that this fracture presented difficulty, and this occurred in twenty-three children who are reviewed here. Results-The result when assessed at two years or more after the injury may be described as good when there is no loss of extension of the elbow and hyperextension is not more than 5 degrees, and there is no change in the carrying angle or loss of more than 10 degrees of flexion. Any arm satisfying these criteria has normal function and appearance when compared with the undamaged limb. 
Of the twenty-three with severely displaced fractures, eight patients had a good result and fifteen showed varying degrees of deformity (Table I).

Analysis-The radiographs after reduction of each case were examined. In every inexact reduction the elements of displacement, angulation and rotation were analysed separately and compared with the final clinical result.

TABLE I

\begin{tabular}{|c|c|c|}
\hline \multicolumn{3}{|c|}{$\begin{array}{l}\text { ANAlysis of the Deformity in FifteEn Patients } \\
\text { Supracondylar Fracture OF the Humerus } \\
\text { (Seven patients had more than one deformity) }\end{array}$} \\
\hline Deformity & $\begin{array}{l}\text { Number of } \\
\text { patients }\end{array}$ & Comments \\
\hline Loss of extension & 2 & $\begin{array}{l}\text { Both had had open } \\
\text { reduction }\end{array}$ \\
\hline Hyperextension . & 6 & All had another deformity \\
\hline Change in carrying angle & 14 & $\begin{array}{l}\text { Of these children, } \\
\text { eight had cubitus varus }\end{array}$ \\
\hline
\end{tabular}

Significance of residual posterior displacement - In eight patients there was persistent posterior displacement of the distal fragment but this did not cause any permanent deformity or disability. An extreme case is illustrated in Figures 1 to 3 , in which serial radiographs showed smoothing of the anterior spike as moulding and growth took place. When reviewed three

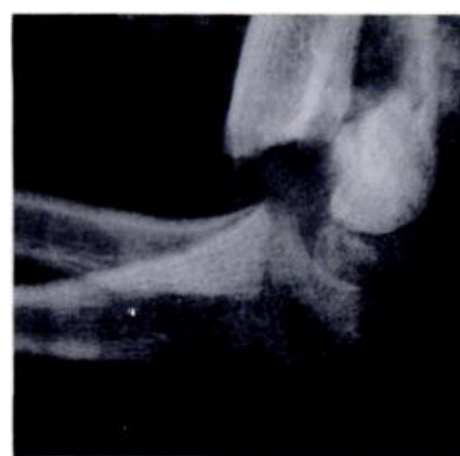

FIG. 1

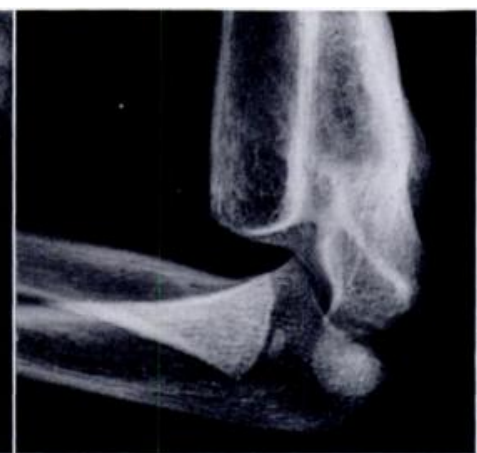

FIG. 2

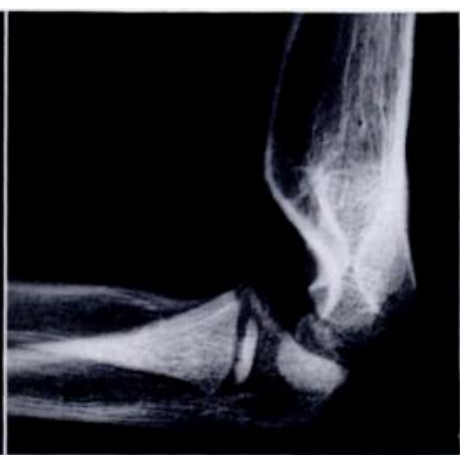

FIG. 3

Case 1-Radiographs showing how moulding took place in an unreduced fracture. Figure 1-Unreduced position, when the child was six years old. Figure 2-Four months later. Figure 3-Three years later.

years later this boy had 7 degrees loss of flexion; no other patient had a measurable loss of flexion.

Significance of medial or lateral displacement-Medial or lateral displacement of the distal fragment did not cause permanent disability, providing there was no associated angulation or rotation. In two patients severe lateral displacement had to be accepted, but there was no residual deformity. Figure 4 is a tracing of a radiograph of a supracondylar fracture in which the medial displacement was not corrected; Figure 5 is a photograph of the patient two years later; the two elbows were identical in appearance and there was no loss of carrying angle.

Significance of posterior angulation-A slight tilt backwards of the distal fragment was common after reduction of even only slightly displaced supracondylar fractures. It is generally thought to cause hyperextension of the elbow.

vol. 45 B, No. 3, AUgust 1963 
Six patients were considered to have 5 degrees or more of hyperextension but only two of them had any posterior tilt of the distal fragment in radiographs after reduction. Five others with a measurable posterior tilt on the radiographs had a normal range of extension at review. The elbow with the greatest hyperextension (11 degrees) had, in fact, been left with some anterior tilt. Thus it is not possible to predict when a hyperextension deformity will occur, and it seems that a small amount of posterior tilt, possibly up to 10 degrees, is quite acceptable.

Significance of medial or lateral angulation-Angulation is difficult to detect by clinical means when the elbow is flexed. On radiographs the deformity is usually not striking and may be accepted unless the position of the fragments is carefully studied. Diagnosis is particularly difficult in a young child before the appearance of the centres of ossification.

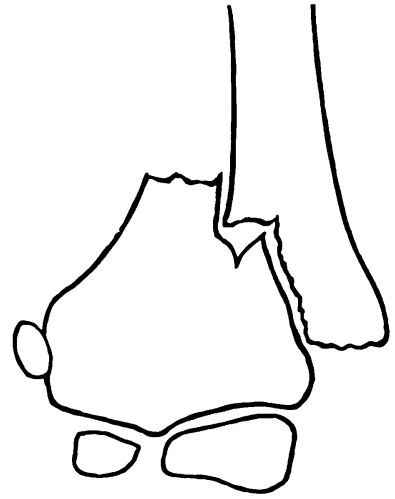

FIG. 4

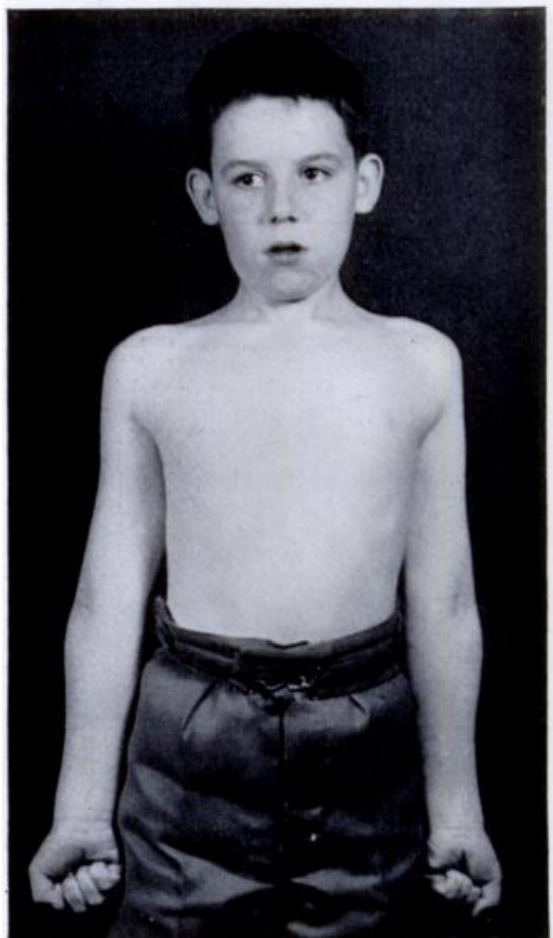

Fig. 5

One patient in this series was left with some valgus tilt of the distal fragment: the result was cubitus valgus of an equal degree. Thirteen patients were left with a varus tilt; the carrying angle of the elbow was diminished in all, and eight had a cubitus varus deformity (Figs. 6 to 9) which did not decrease with the passage of time, even in the youngest group (three children were less than three years of age at the time of injury). There can be no doubt that varus or valgus angulation causes a change in the carrying angle which is not corrected by growth or moulding.

Significance of rotation-Medial rotation of the distal fragment is a significant feature of the original displacement in most supracondylar fractures. Lateral rotation is uncommon. When the fracture is incompletely reduced there often persists an element of rotation which may only be detected by a careful study of radiographs.

Of eighteen inaccurate reductions ten had residual medial rotation, always in association with a further element of displacement or angulation. Seven were associated with medial angulation, all showed a diminution of the carrying angle and four developed cubitus varus. 


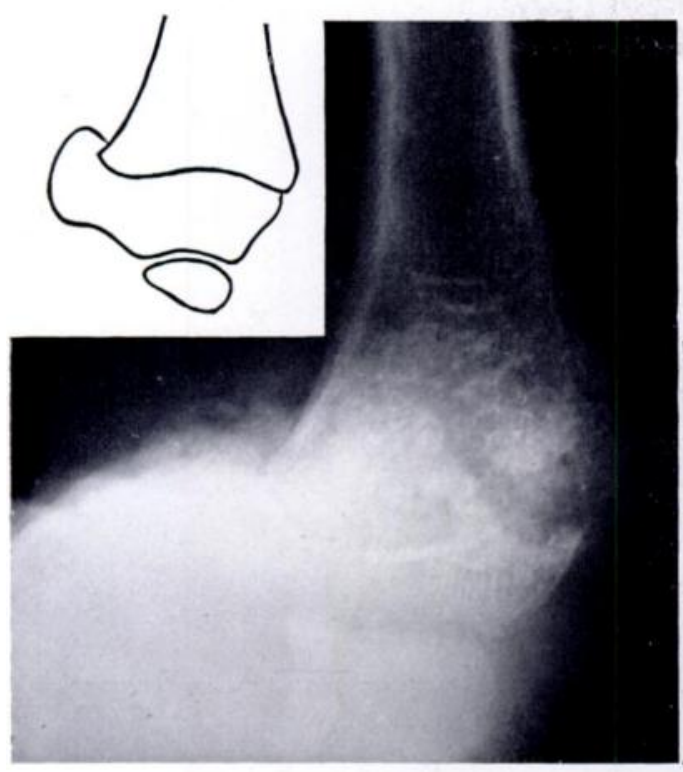

Fig. 6

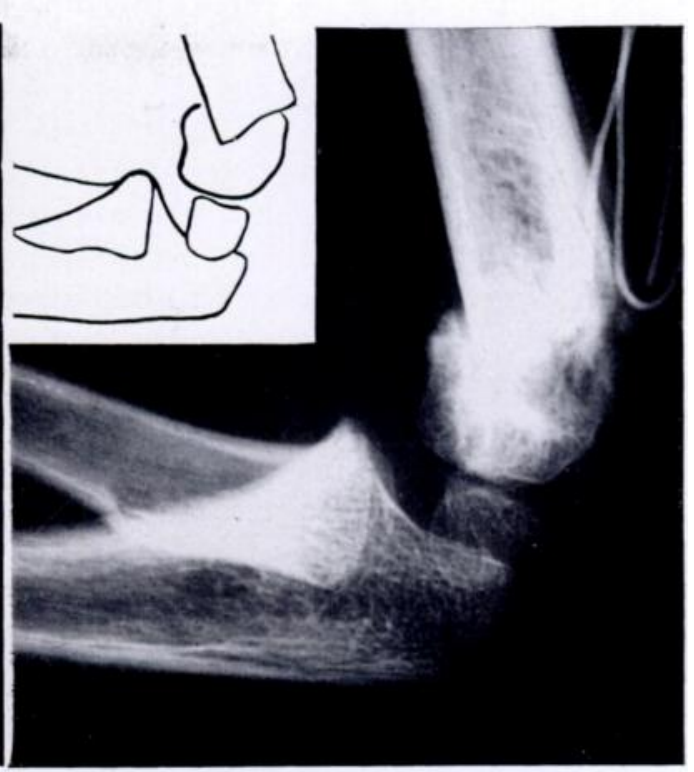

Fig. 7

Case 3-Radiographs after reduction in a child who developed cubitus varus. The distal fragment is angulated medially, but there is no rotation.

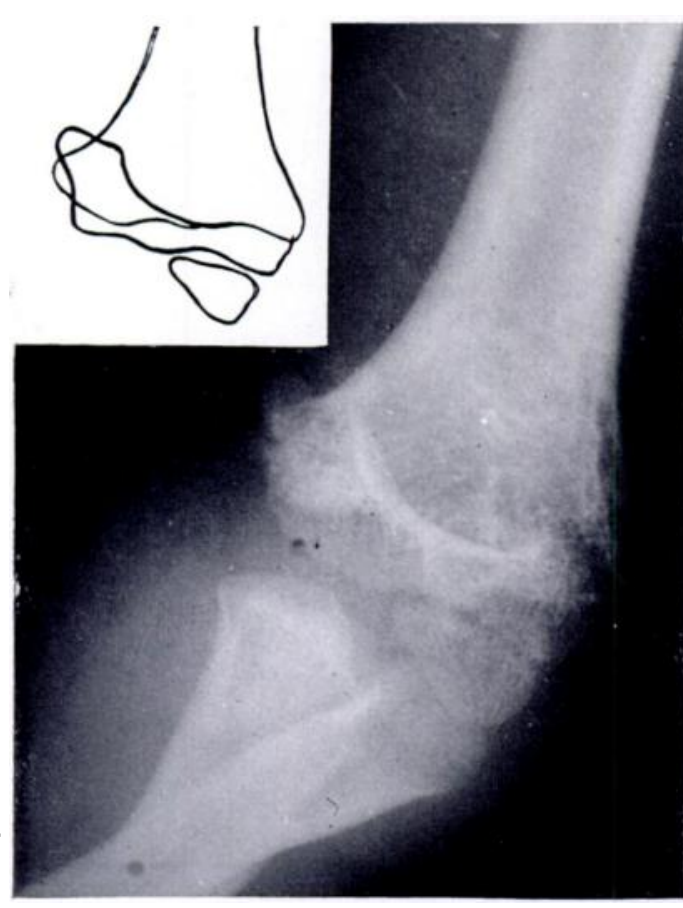

FIG. 8

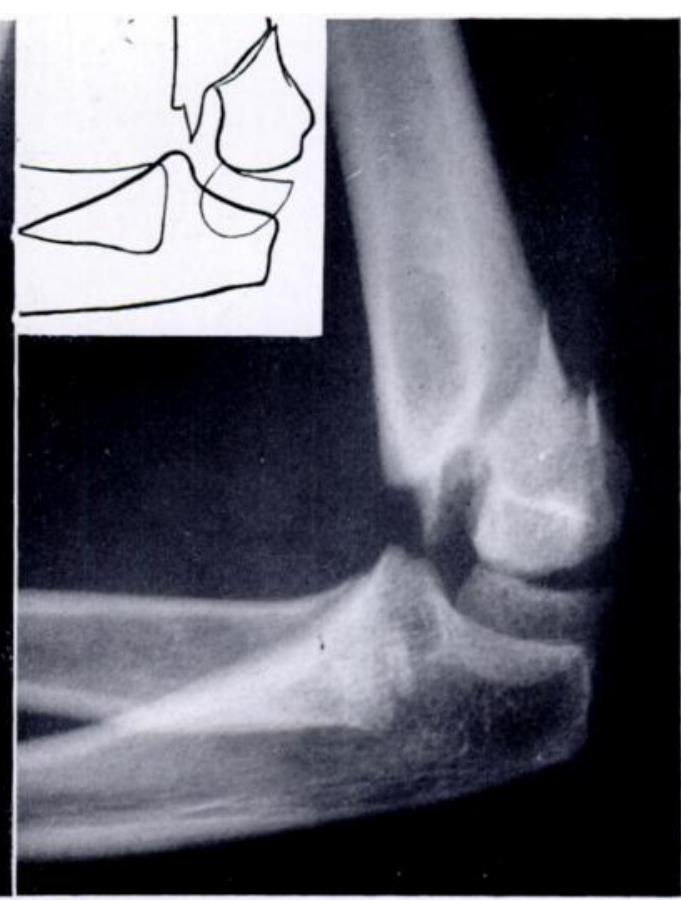

FIG. 9

Case 4-Another child who developed cubitus varus. The radiographs show the medial angulation and the rotation.

vol. 45 B, No. 3, AUGUST 1963 
In two patients medial rotation was associated with posterior or lateral displacement of the distal fragment, and in one patient with a posterior tilt: the final result in each was a normal elbow. These findings indicate that the varus deformity is attributable to the medial angulation and not to medial rotation.

Open operation-Five patients had an open operation, one because the fracture was open (compound), one because the brachial artery became obstructed and three to secure reduction. Figure 10 illustrates a patient in whom open operation was carried out because of instability of the fragments, and an anatomical and stable reduction was obtained without internal fixation. The elbow now lacks 55 degrees of extension because of contracture of the soft tissues in front of the joint; there has been no improvement during the last two years. The child with the open supracondylar fracture also lost 18 degrees of extension of the elbow joint.

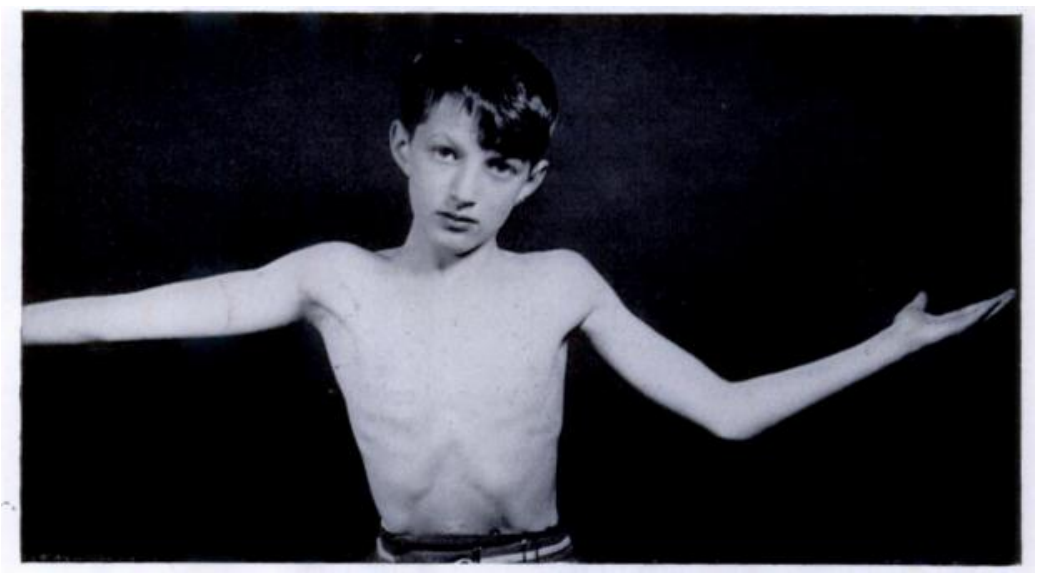

FIG. 10

Case 5-Three years before, an open reduction had been done on this boy's left elbow because the bony fragments were unstable; an anatomical reduction was obtained. There is a permanent loss of extension of 55 degrees.

\section{DISCUSSION AND CONCLUSIONS}

There are three significant complications of supracondylar fracture of the humerus: ischaemic contracture, loss of extension at the elbow and cubitus varus.

Volkmann's ischaemic contracture is the most serious complication and leads to a maimed limb. It follows impairment of circulation in the arm and is not a product of inaccurate reduction. Loss of extension causes disfigurement and impairment of function. A supracondylar fracture does not involve the articular surfaces and should not lead to limitation of extension: when this occurs it is usually due to damage to the soft tissues in front of the joint. Since the recognition of the part played by passive exercises in the origin of myositis ossificans almost all arms treated by closed manipulation have regained normal extension (Siris 1939).

The advocates of open reduction appear to accept limitation of extension as a mild disability: patients, and their parents, hold a different view. In one of the largest series treated by open reduction (Sandegaard 1943) thirteen of the seventy-nine patients had limitation of extension of the elbow. Similarly, many patients with open fractures have a residual flexion contracture from the severe damage to soft tissues.

Operation is obligatory when the fracture is open or when there is vascular involvement. As an elective procedure it can be justified only if the results are better than those of closed reduction. The bad results of manipulation, caused by angulation of the fragments, are no easier to avoid when the fracture is treated by open reduction.

Cubitus varus is generally accepted as being the commonest deformity after supracondylar fracture. Most critical authors report that 50 to 60 per cent of severely displaced supracondylar 


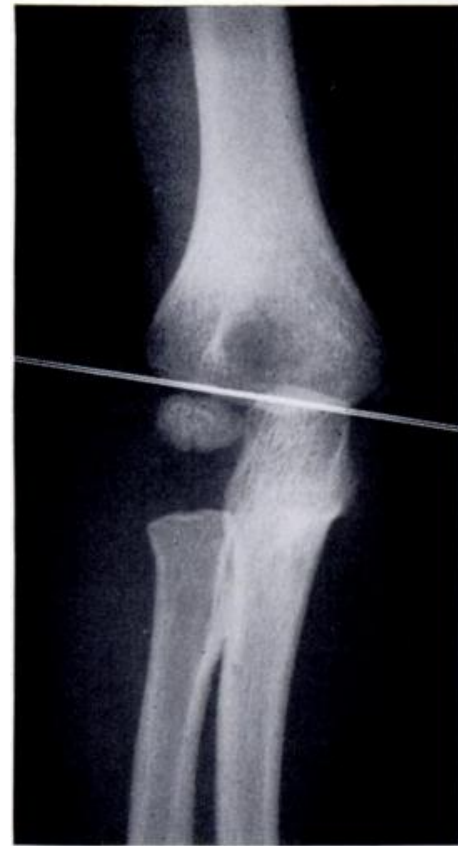

FiG. 11

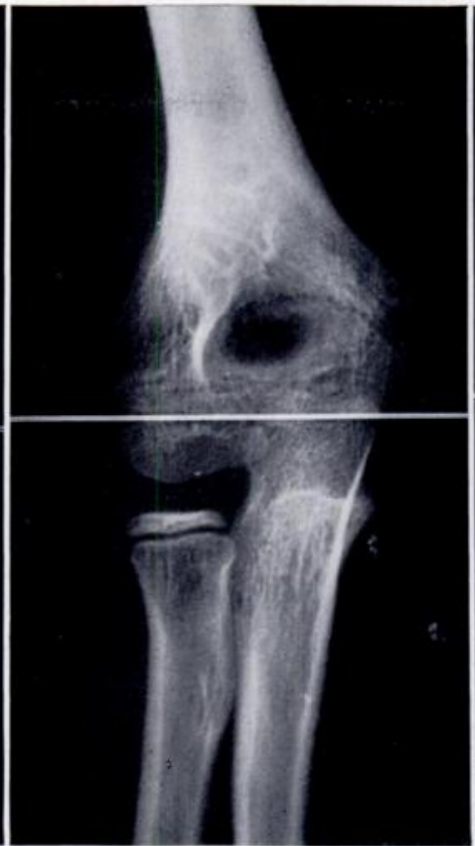

FIG. 12

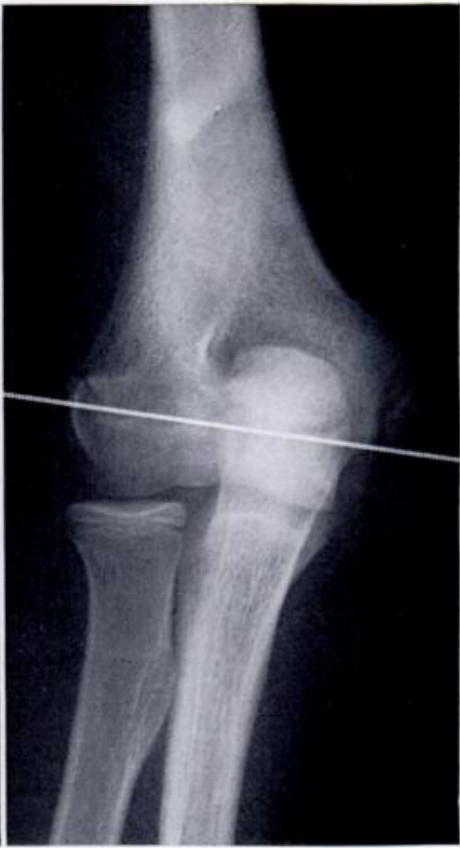

FIG. 13

Radiographs of normal elbows showing the appearance of the lower epiphysis. Figure 11-At the age of four years. Figure 12-At the age of nine years. Figure 13-At the age of fifteen years. At all ages the epiphysial line is perpendicular to the ulna, not the line of the humerus. The centre for the medial epicondyle is entirely separate from the main epiphysis.

fractures have alteration of the carrying angle and at least 20 per cent show a cubitus varus deformity. Elaborate fixation techniques do not seem to improve these proportions.

Cubitus varus causes disfigurement rather than loss of function. It is the only severe deformity which may be found after an otherwise uncomplicated closed manipulation and is caused by medial angulation (varus tilt) of the distal fragment.

Radiographs after reduction often reveal uncorrected medial rotation, but there is no evidence that it is responsible for a reduction of the carrying angle. The significance of medial rotation is that there is no contact on the medial side between the cortex of the distal fragment and the cortex of the shaft of the humerus. Consequently the distal fragment may tilt medially; this may occur either at the time of manipulation or during the succeeding days before the fracture achieves stability. Deformity is the result of this secondary angulation and does not occur in its absence.

Simple displacement without rotation or angulation of the distal fragment does not cause any alteration of the carrying angle. Even a poor reduction will produce a normal elbow if the correct alignment is maintained (Figs. 4 and 5).

A fracture may unite with a varus tilt when the surgeon is unable to correct alignment, or because he does not appreciate that he has failed to correct alignment, or because the reduction may be unstable or fixation inadequate. It is the thesis of this paper that cubitus varus is predictable but not necessarily preventable. Nevertheless, fewer cases will occur if the radiographs are studied critically.

Interpretation of radiographs-Most supracondylar fractures occur between the ages of two and ten years, after the appearance of the epiphysis for the capitulum but before the trochlear centre has fully developed. Thus interpretation of radiographs after reduction can be difficult, and it may be advisable to obtain views of the normal elbow before any procedure is attempted. In this fracture it is much more important to correct alignment than a slight degree of displacement, but the latter is more apparent on a radiograph. 
Proper interpretation demands a discriminating eye and an ability to analyse the third dimension. Certain features are worthy of emphasis: 1) The positions of the radius and ulna must be ignored. When the elbow is flexed or the forearm pronated no measure of the carrying angle can be made. 2) The radio-opaque lower end of the humerus does not indicate the plane of the elbow joint until the epiphysial centres are fully developed. 3) The epiphysial line is not at right angles to the shaft of the humerus: it is parallel to the elbow joint and at right angles to the true line of the forearm (Figs. 11 to 13). 4) The lower part of the shaft of the humerus is flat in section. Medial rotation is indicated by the presence of a "spike" or by apparent disparity in the diameters of the broken bone ends (Fig. 9). 5) Small but significant degrees of angulation may be detected by comparison of the apposition obtained at the medial and lateral aspects of the cortex (Figs. 6 and 8).

Procedure-It is concluded that the uncomplicated fracture is best treated by closed manipulation under radiographic control. The surgeon must be experienced and prepared to take responsibility for the final position. The decision to accept an imperfect reduction should be taken only after full consideration of the effect any residual displacement will have on the appearance and function of the arm. The method of fixation will depend upon personal preference and an assessment of the stability of the fragments: slipping is more likely to occur if medial rotation has not been fully corrected.

When the reduction has been inexact the only significant deformity to be expected is cubitus varus. When an experienced surgeon has failed to achieve alignment of the fragments the child should not be subjected to further anaesthesia or open reduction. The outlook is good and there will be no significant loss of function.

\section{SUMMARY}

1. During two years eighty-five children with supracondylar fractures of the humerus were admitted to a children's hospital. This paper is a study of the severe fractures which occurred in twenty-three children of whom fifteen had a deformity at review.

2. The only deformity found after treatment by closed manipulation was an alteration in the carrying angle. It is considered that cubitus varus was caused by medial angulation of the distal fragment.

I wish to thank Professor Roland Barnes and Mr N. J. Blockey for reading and criticising the manuscript of this paper. I am indebted to Mr Blockey and to Mr D. A. Macpherson for allowing me to study patients under their care. I am grateful to the Departments of Medical Illustration at the Western Infirmary and at the Royal Hospital for Sick Children, Glasgow, for the drawings and photographs.

\section{REFERENCES}

Attenborough, C. G. (1953): Remodelling of the Humerus after Supracondylar Fractures in Childhood. Journal of Bone and Joint Surgery, 35-B, 386.

Brewster, A. H., and KarP, M. (1940): Fractures in the Region of the Elbow in Children. Surgery, Gynecology and Obstetrics, 71, 643.

French, P. R. (1959): Varus Deformity of the Elbow. Lancet, ii, 439.

HøYer, A. (1952): Treatment of Supracondylar Fracture of the Humerus by Skeletal Traction in an Abduction Splint. Journal of Bone and Joint Surgery, 34-A, 623.

KING, D., and SeCor, C. (1951): Bow Elbow (Cubitus Varus). Journal of Bone and Joint Surgery, 33-A, 572. LAURENCE, W. (1956): Supracondylar Fractures of the Humerus in Children. British Journal of Surgery, 44, 143.

MADSEN, E. (1955): Supracondylar Fractures of the Humerus in Children. Journal of Bone and Joint Surgery, 37-B, 241

SANDegaARD, E. (1943): Fractures of the Lower End of the Humerus in Children. Acta Chirurgica Scandinavica, 89, 1.

Siris, I. E. (1939): Supracondylar Fracture of the Humerus. Surgery, Gynecology and Obstetrics, 68, 201.

Smith, L. (1960): Deformity Following Supracondylar Fractures of the Humerus. Journal of Bone and Joint Surgery, 42-A, 235. 